\title{
La Educación Emocional como eje vertebrador de la pedagogía preventiva o previa a la muerte
}

Emotional Education as a vertebrating axis of preventive or pre-death pedagogy

Anabel Ramos-Pla ${ }^{1}$ Universidad de Lleida

Recibido: 21.08.2021

Aceptado: 20.10.2021

\section{Resumen}

Esta ponencia se fundamenta en la innegable y estrecha relación existente entre la Educación Emocional y la pedagogía preventiva o previa a la muerte que se puede llevar a cabo en las escuelas, más necesaria si cabe a raíz de la pandemia por la COVID-19. A partir de las bases que se exponen, se interpelan los conceptos para ensalzar la vinculación pedagógica y didáctica existente entre la Educación Emocional y la Pedagogía preventiva o previa a la muerte dentro de los centros educativos. A través del metaanálisis y de la reflexión conceptual, se expone la importancia de la incorporación y relación de los dos ámbitos de conocimiento con los que se pueden desarrollar multitud de beneficios como son el fomento de la Inteligencia Emocional en el alumnado de los diferentes centros educativos. Por último, se proponen diferentes estrategias, herramientas, tareas y actividades útiles y funcionales que relacionan la Educación Emocional con la Pedagogía preventiva o previa a la muerte para llevar a cabo en las escuelas o institutos.

\footnotetext{
1 anabel.ramos@udl.cat https://orcid.org/0000-0001-5437-150X
} 
Palabras clave: educación para la muerte, inteligencia emocional, duelo, educación primaria, aprendizaje socioemocional.

\begin{abstract}
This paper is based on the undeniable and close relationship between Emotional Education and the preventive or pre-death pedagogy that can be carried out in schools, which is even more necessary in the wake of the COVID-19 pandemic. On the basis of the bases presented, the concepts are questioned in order to highlight the existing pedagogical and didactic link between Emotional Education and preventive or pre-death pedagogy in educational centres. Through meta-analysis and conceptual reflection, the importance of incorporating and relating the two fields of knowledge with which a multitude of benefits can be developed, such as the promotion of Emotional Intelligence in the students of the different educational centres, is presented. Finally, different strategies, tools, tasks and useful and functional activities are proposed that relate Emotional Education with preventive or pre-death pedagogy to be carried out in schools or institutes.
\end{abstract}

Keywords: death education, emotional intelligence, grief, primary education, social emotional learning 


\section{Introducción}

La muerte supone todavía un tabú social (Herrán, 2015, 2017; Peniston, 2015; Ransanz, 2015; Corr, 2016; Gill, 2016; Park, Lee, Kim \& Smith-Stonen, 2016; Yang \& Park, 2017; Chau, Cheung \& Ho, 2018; Martínez-Heredia \& Bedmar, 2020, Ramos-Pla, 2020aentre otros) que contamina el ámbito pedagógico de forma imperante, aunque seguimos sumergidos en la pandemia por la COVID-19 (hecho que ha acelerado la muerte de muchas personas) (RamosPla, 2020d). De este modo, aunque cada vez más se van ampliando las investigaciones en relación con la pedagogía de la muerte, continúan siendo escasos los estudios en relación a la pedagogía preventiva o previa a la muerte (Ramos-Pla \& Gairín, 2020; Ramos-Pla, Gairín \& Camats, 2020).

Sin embargo, un tema que se ha investigado mucho a lo largo del siglo XXI es la Educación Emocional. Por ello, con este artículo se construyen vínculos en torno a la pedagogía preventiva o previa a la muerte con la Educación Emocional (EE) y, yendo más allá, con la Inteligencia Emocional (IE). Es necesaria elaborar dichas relaciones, ya que mediante un programa de EE se pueden trabajar las emociones que desarrollan la muerte de forma previa al trágico acontecimiento. Así, se puede tratar la muerte y sus emociones de forma preventiva, tratando de evitar futuros duelos patológicos (Morell-Velasco et al., 2018).

Por último, se presenta la relación entre EE, creatividad y pedagogía preventiva o previa a la muerte. Con ello se pretende aprovechar un hecho vital para el desarrollo de competencias creativas en el alumnado. 


\section{Metodología}

El método utilizado para obtener la bibliografía citada en el presente estudio sobre la necesidad de una pedagogía de la muerte se ha obtenido a partir de diversas bases de datos nacionales (Dialnet, RESH, Psicodoc, REDINED y repositorios institucionales de universidades españolas) así como internacionales (Scopus, Web of Science, ERIC, IRESIE, Latindex, WorldCat, Sudoc y Scielo). Teniendo en cuenta lo anterior, se llevó a cabo un seguimiento y análisis sistemático de las publicaciones realizadas en los últimos 7 años.

Sin embargo, se debe tener en cuenta que se utilizaron criterios como la importancia de la autoría (poniendo énfasis en los autores pioneros en la temática y en aquellos que asentaron los fundamentos de la misma), la calidad científica y la idoneidad con la pedagogía preventiva o previa a la muerte. Este último criterio alberga gran importancia ya que, aunque cada vez más se extiende la producción científica sobre la temática que nos ocupa, todavía sigue siendo un tabú social y pedagógico, hecho que dificulta la cantidad de artículos analizados.

\section{La pedagogía preventiva o previa a la muerte}

La pedagogía preventiva sobre la muerte se centra en la educación de esta, teniendo como base la normalización de la muerte (Cortina, Herrán \& Nolla, 2009; Girardi, San Gil \& Santillán, 2009; Poch, 2009; Asensi, 2012; Benlloch, Vazquez, Boluda \& Garcia Bataller, 2015; Head \& Smith, 2016; Gao \& Slaven, 2017; Campillo Ruiz \& Ruiz Arriaza, 2017; Colomo \& Cívico, 2018; Ramos-Pla, 2017) para favorecer la formación integral de los educandos. En este sentido, compartimos el argumento de Huisman-Perrin (2003, p.11) quien entiende el diálogo con la muerte desde una perspectiva en la que se debe "considerarla sin empequeñecerla, mesurarla sin minimizarla, evocarla con precisión sin dilucidarla no es ni entenderla ni explicarla propiamente dicho, tareas racionalmente imposibles, sino que es escucharla o domesticarla para evitar el horror y el miedo". 
Es necesaria la toma de conciencia sobre la finitud humana, ya que da tiempo para cuestionarse y reflexionar sobre todo aquello que se quiere hacer durante el tiempo que se dispone (Poch, 2009), mientras se reconsidera -o considera por primera vez- la vida. A su vez, se favorece la superación positiva de las pérdidas que se hayan sufrido a lo largo de la vida (Colomo, 2016).

Entendemos por finitud la definición realizada por Mèlich (2011, p.15) quién afirmó que somos seres que estamos realizando un trayecto vital hasta de un espacio y tiempo y, añade que "la finitud es sinónimo de vida". El filósofo afirma que es necesaria la pedagogía preventiva o previa a la muerte, y en esto estamos completamente de acuerdo. De este modo, apostamos por el aprendizaje del tiempo y no solo en el tiempo. Por lo tanto, siguiendo con el hilo de la definición de finitud, la muerte es el final del trayecto de la vida. Mèlich (2011) entiende la muerte como condicionante de la finitud; no como parte de la vida. Sin embargo, nos contraponemos en parte a la tesis de Mèlich, dado que la muerte es condicionante de la finitud porque forma parte de la vida. Si no formara parte de la vida, la muerte no acontecería ningún tipo de condicionante de la finitud. Además, autores como Kübler-Ross, apuestan por una definición de la muerte como "experiencia única, bella, liberadora, que se vive sin temor y sin angustia" (1992, p.16). Hay que añadir que, para realizar esta definición de la muerte, la autora citada tuvo muchas experiencias como psiquiatra con enfermos terminales. La doctora los enseñaba a cerrar asuntos pendientes que tuvieran en vida para aceptar su muerte próxima y, así, poder morir en paz. Si no se van cerrando progresivamente los asuntos pendientes que se vayan teniendo a lo largo del transcurso de la vida es imposible vivir plenamente. Por este motivo, pensamos que enseñar que la muerte es una experiencia vital de la vida, puede ayudar a aceptar de forma más normalizada la propia defunción o la de los seres queridos.

Mediante la pedagogía preventiva o previa a la muerte se pretende tratar la muerte con normalidad, hablando sobre ella anticipadamente para poder crecer internamente y, así, poder estar preparados para su llegada (Kübler-Ross, 1992; Mantegazza, 2006; Girardi, San Gil \& Santillán, 2009; Valenzano, 2015). De esta forma, pretendemos prepararnos en el sentido 
existencial, mediante la toma de conciencia de la existencia de la muerte. En consecuencia, nos estaremos preparando para sufrir el duelo de forma menos traumática y patológica (Aradilla, 2004; Rodríguez Herrero, Herrán \& Cortina, 2015).

El hecho de conocer las emociones y los hechos que desencadena la muerte de un ser querido posibilita una elaboración del duelo más positivo. A la vez, mediante esta normalización se pretende que los niños vayan perdiendo progresivamente el miedo en la muerte, aceptarla como una realidad inevitable de los seres vivos mediante su deconstrucción conceptual (Poch, 2009; Comas \& Carmelo, 2014) para, así, potenciar la concepción de la muerte como una herramienta posibilitadora de crecimiento personal, dado que puede acontecer la brújula que defina el sentido que se quiera dar a la vida.

\section{Educación Emocional en la pedagogía preventiva o previa a la muerte}

Actualmente, la Educación Emocional está en auge en el mundo educativo (sobre todo en Educación Infantil y Educación Primaria, a pesar de que puede desarrollarse desde todos los niveles), ya que representa una forma de prevención primaria (Bisquerra, 2003, 2006). Los profesionales de la educación y de la psicología han potenciado la toma de conciencia que el aprendizaje se vincula a las emociones experienciadas, que se configuran y aprenden a lo largo de la vida (Gómez Ruiz, 2013; Ramos-Pla, 2020b)). Masreal (2007) lo describe des la vertiente psicológica en cuanto que la información que llega al cerebro para ser analizada pasa antes por el sistema límbico, donde se encuentran situadas las emociones. Es decir, primero actúa la emoción emitiendo un filtraje y después la razón. La etimología de la palabra "emoción" nos rebela el mismo significado, dado que "moción" significa sentimientos y "e" quiere decir hacia "fuera". Cabe añadir que las habilidades emocionales son susceptibles de aprendizaje y perfeccionamiento a lo largo de la vida.

Tener una buena gestión de las emociones es esencial para obtener una satisfacción personal y una buena relación con los que nos rodean (Griscom, 2000; Guil, Gil-Olarte, Mestre \& Nuñez, 2006). Al fin y al cabo, es el que desde hace unos años se denomina Inteligencia 
Emocional (Salovey \& Mayer, 1990, Goleman, 1995; Extremera \& Fernández-Berrocal, 2004; Mestre Navas \& Fernández Berrocal, 2007). En esta inteligencia se engloba la relación entra la cognición y la emoción. Desde un principio Salovey y Mayer (1990) introdujeron el concepto de IE como "la habilidad para percibir emocionas; para acceder y generar emocionas que facilitan el pensamiento; para comprender emocionas y conocimiento emocional, y para de forma reflexiva regular emocionas que promuevan tanto el crecimiento personal como intelectual". En definitiva, esta definición se refiere a la capacidad de gestionar emociones para canalizarlas de forma constructiva y positiva.

El motivo clave por el cual tratamos la Educación Emocional es la contribución que aporta en la educación integral de los niños y las niñas, dado que estamos de acuerdo con Torralba (2008) en afirmar que, si la Educación Emocional no se integra en la educación y no hay ningún tipo de vínculo afectivo entre el educador y el alumnado, no se realizará una educación integral. En la tabla 1 se pueden observar las diferentes habilidades que se desprenden del modelo de Inteligencia Emocional.

Tabla 1. Modelo de Inteligencia Emocional.

\begin{tabular}{|c|c|}
\hline Percepción, evaluación y expresión de las emociones \\
\hline - & Habilidad para identificar emociones de nuestros estados físicos y psicológicos. \\
- & Habilidad para identificar emociones en otras personas u objetos. \\
- & Habilidad para expresar emociones con seguridad y expresar las necesidades relacionadas a \\
- & aquellos sentimientos. \\
& Habilidad para discriminar la expresión de emociones honestas y deshonestas, exactas o \\
\hline Facilitación emocional del pensamiento & Habilidad para redirigir y priorizar nuestro pensamiento basado en los sentimientos asociados a \\
& objetos, acontecimientos u otras personas. \\
- & Habilidad para generar y revivir emociones con el fin de facilitar juicios o recuerdos. \\
- & Habilidad para capitalizar las oscilaciones emocionales para tomar múltiples puntos de vista; \\
& habilidad para integrar aquellas perspectivas inducidas por nuestros sentimientos. \\
- & Habilidad para utilizar los estados emocionales para facilitar la solución de problemas y la \\
& creatividad.
\end{tabular}




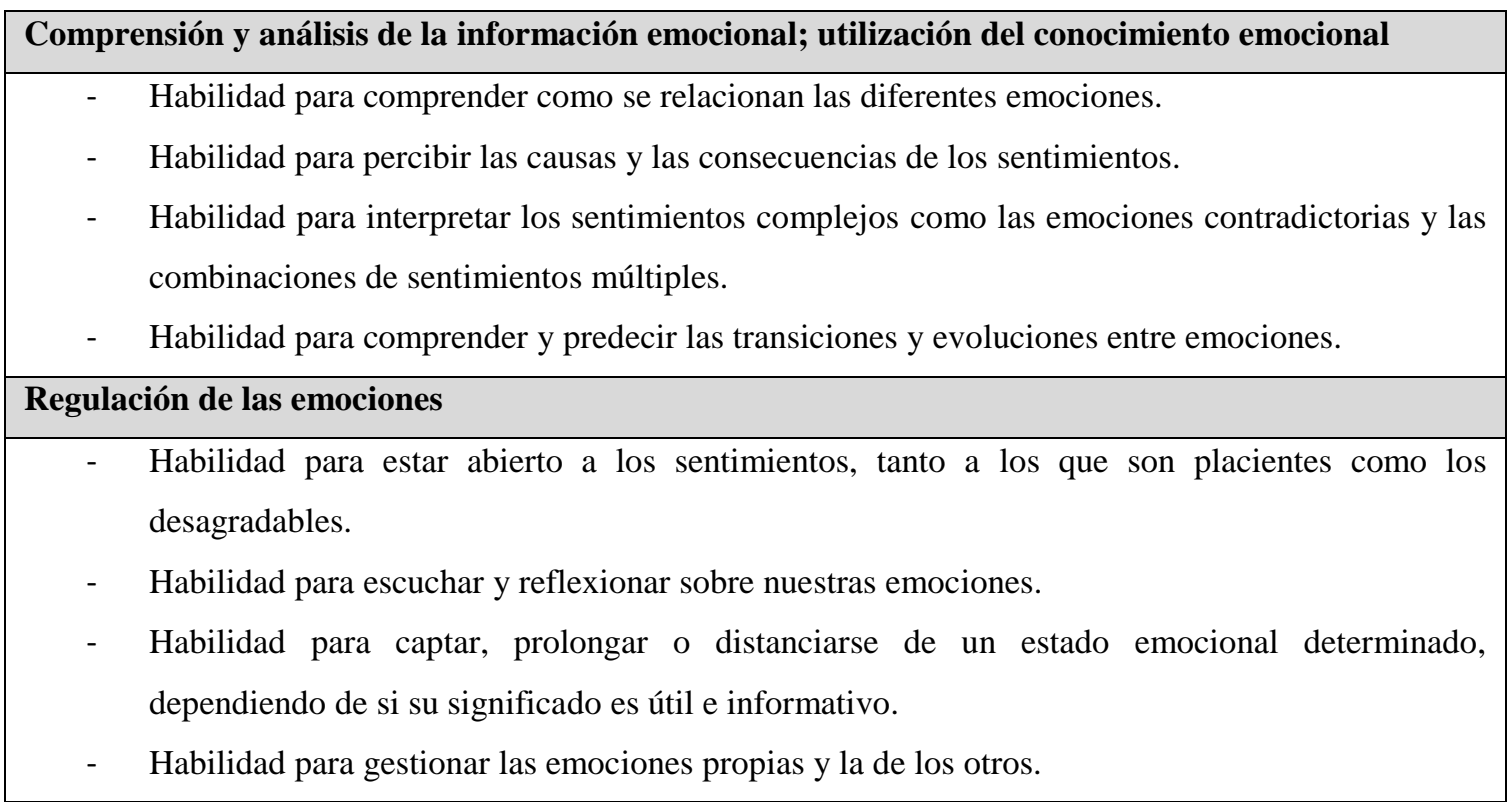

Tabla adaptada de Salovey, Mayer \& Caruso (2008), Mestre Navas \& Fernández Berrocal (2007), Extremera \& Fernández-Berrocal (2003: 101), Vallés \& Vallés (2003).

La Educación Emocional es importante para acompañar en el desencadenamiento de reacciones emocionales que comporta el duelo por la muerte de una figura de apego (Dollenz, 2003, Pedrero-García, 2020). En definitiva, la EE sirve para gestionar todos los sentimientos (desarrollados en apartados anteriores) que desencadena la muerte de un ser querido, dado que la regulación emocional legitima las emociones adecuadamente.

Debemos recordar que Gardner (2010) reformuló la teoría de la inteligencia para identificar ocho inteligencias. El psicólogo era crítico con la concepción tradicional de la inteligencia, de la cual se pensaba que solo había de un tipo y decidió hacer una reformulación de la teoría. De las ocho inteligencias que propone, Gardner, destaca dos tipologías de inteligencia personal que tienen una dependencia mutua (Salovey, Mayer \& Caruso 2008; Darder \& Bach, 2006): interpersonal (permite las relaciones y a comprensión de los otros) y la interpersonal (configuración de la imagen propia). De esta forma, tal y como apelan Extremera y Fernández-Berrocal (2004, p.6): "Las personas emocionalmente inteligentes no solo serán hábiles para percibir, comprender y gestionar sus emociones, sino también serán más capaces de extrapolar sus habilidades de percepción, comprensión y gestión a las emociones 
de los otros". Posteriormente, Salovey y Mayer (1990) a partir de las primeras publicaciones de Gardner, organizó las inteligencias personales en cinco grandes competencias: conocimiento propio de las emociones y capacidad de controlarlas; capacidad de automotivación, reconocimiento de las emociones ajenas y control de las relaciones.

Diversos estudios relacionan la IE con los niveles de bienestar y ajuste emocional (Bisquerra, 2003; Guil, Gil-Olarte, Mestre \& Nuñez, 2006; Latorre \& Montañés, 2004; Lantieri, 2009; Salovey, Mayer \& Caruso, 2008). En estos estudios se estima la correlación positiva significativa entre el ajuste psicológico y el bienestar. Los mismos autores apelan al hecho que las personas que disponen de una inteligencia emocional más desarrollada, tienden a tener menos estados psicológicos de base negativa y desarrollan un mayor sentido de adaptabilidad y flexibilidad a los continuos cambios; a la vez que actúan como protección de los efectos burnout que sufren algunos docentes (Lantieri, 2009). En este sentido, destacamos los estudios realizados por Guil, Gil-Olarte, Mestre \& Nuñez (2006) quienes estudiaron la correlación entre los resultados académicos y la Educación Emocional con una muestra de 77 individuos de $4 .^{\circ}$ de ESO de Cádiz. Los resultados mostraron que los alumnos que obtenían mejores notas tenían niveles más altos de competencia social y adaptabilidad. A través de este estudio, los autores concluyeron que un entrenamiento en competencias socioemocionales repercute positivamente en las notas académicas del alumnado y en la adaptabilidad.

Conviene destacar los estudios realizados por Latorre y Montañés (2004), Ramos-Pla y Selva (2020) y Ramos-Pla (2020e), quienes analizaron la relación entre la IE y las conductas saludables, junto con las percepciones de la muestra de población (462 alumnos de bachillerato y primer curso de universidad de Albacete). Los resultados resaltaron la inexistencia de relación directa entre la IE y las conductas saludables. Pero, aparece una relación negativa entre percepción de la propia salud y bienestar y los comportamientos más saludables, incluyendo un menor consumo de alcohol y drogas.

Teniendo en cuenta el marco citado, donde se resalta la importancia de trabajar una Educación Emocional con el alumnado, cabe destacar que dentro de la misma existen una serie de 
objetivos o metas y competencias. A grandes rasgos, el modelo que plantea Goleman (1995) de Inteligencia Emocional a desarrollar mediante una EE, contiene las siguientes metas:

. Conocer las propias emociones.

. Gestionar las emociones para expresarse de forma adecuada.

. Motivarse uno/a mismo/a para ser más productivo/a y efectivo/a.

. Reconocer las emociones de los demás.

. Establecer relaciones.

Goleman (1995) se basó en el modelo de Salovey \& Mayer (Bisquerra, 2003; Vallés \& Vallés, 2003) y distingue en el modelo de competencias emocionales un conjunto de habilidades, donde destacan el autocontrol, el entusiasmo, la empatía, la perseverancia y la capacidad por automotivarse. Si las emociones se canalizan y expresan correctamente, se pueden apaciguar situaciones de ansiedad y estrés, buscar soluciones creativas por emociones negativas y empatizar con los otros (Extremera, \& Fernández-Berrocal, 2004; Mestre Navas \& Fernández Berrocal, 2007). En la tabla 2 se puede observar las relaciones existentes entre las competencias emocionales (plateadas por diversos autores) y unas nociones sobre su relación con la muerte (y las emociones que se le relacionan).

Tabla 2. Definición de las competencias emocionales y su relación con la muerte.

\begin{tabular}{l}
\hline Competencia de conciencia emocional \\
Consiste en conocer las propias emociones y las de los otros. En este bloque, se pueden encontrar las \\
siguientes microcompetencias: \\
- $\quad$ Toma de conciencia de las propias emociones. \\
- $\quad$ Asignación de nombre a las emociones. \\
- Comprensión de las emociones propias y de los otros. \\
- Toma de conciencia de la interacción entre emoción, cognición y comportamiento. \\
Relación con la muerte: Toma de conciencia de las emociones que se desarrollan ante la muerte de un ser \\
querido; identificarlas, ponerles nombre, detectarlas y comprender la de los demás. \\
Competencia de regulación emocional \\
\hline Es la capacidad para gestionar las emociones de forma adecuada. Se pueden encontrar las \\
siguientes microcompetencias:
\end{tabular}


- $\quad$ Expresión emocional de forma apropiada.

- Regulación emocional.

- Habilidades de afrontamiento.

- Autogeneración de emociones positivas.

Relación con la muerte: Regulación y gestión las propias emociones para desarrollar estrategias de afrontamiento a la muerte.

\section{Competencia de autonomía emocional}

Es un concepto amplio que incluye elementos interrelacionados como la autogestión personal y la autoeficacia emocional. En este sentido, se pueden encontrar las siguientes microcompetencias:

- Autoestima.

- Automotivación.

- Autoeficacia emocional.

- Responsabilidad.

- Actitud positiva.

- Análisis crítico de normas sociales.

- Resiliencia.

Relación con la muerte: Enfrentar la muerte de un ser querido.

\section{Competencia social}

Capacidad para mantener relaciones positivas con otras personas. Las microcompetencias que la componen son las siguientes:

- Dominio de las habilidades sociales básicas.

- Respeto por los demás.

- Práctica de la comunicación receptiva.

- Práctica de la comunicación expresiva.

- Capacidad de compartir emociones.

- Comportamiento prosocial y cooperación.

- Asertividad.

- Prevención y resolución de conflictos.

- Capacidad para gestionar situaciones emocionales.

Relación con la muerte: capacidad de ponerse al lugar del otro (empatía) cuando ha sufrido una pérdida de un ser querido, entender aquello que siente y acompañarlo en el proceso de duelo. Permitir que la persona en duelo exprese sus emociones y legitimarlas.

\section{Competencias para la vida y el bienestar}

Es la capacidad para adoptar conductas adecuadas y responsables para hacer frente satisfactoriamente a los retos que se plantean a lo largo de la vida. Las microcompetencias que la conforman son: 
- Fijación de objetivos adaptativos.

- Toma de decisiones.

- Búsqueda de ayuda y recursos.

- Bienestar emocional.

- Capacidad de fluir en el porvenir.

Relación con la muerte: El conjunto de competencias emocionales anteriores relacionadas con la muerte permiten que se acabe consiguiendo la competencia para la vida y el bienestar teniendo en cuenta la muerte como un hecho normalizado e inevitable.

Tabla adaptada de Goleman (1995), Vallés \&Vallés (2003), Bisquerra (2003, 2006, 2016).

Kübler-Ross (1992) afirmó que el ser humano dispone de cinco estados emocionales naturales altamente relacionados con la muerte que se tienden a tergiversar:

. Miedo. Solo se nace con dos miedos inherentes: caer desde muy alto y a escuchar, de repente, ruidos muy fuertes. Es decir, los humanos nacemos con estos miedos, pero durante el transcurso de la vida, las tergiversamos. Por ejemplo, estos dos miedos acaban derivando en emociones desfiguradas como: miedo al fracaso, al rechazo, al triunfo, a la violencia, a la depresión, etc.

- Área emocional. Los infantes sienten miedo a los dos factores mencionados en el punto anterior, pero no a la muerte. Aun así, sí que tienen miedo a la separación, sobre todo a la de la madre y/o el padre, dado que para ellos/a es esencial que no los abandonen y que los cuiden.

- Compartir las emociones. Cuidar de los familiares que están enfermos puede ser una experiencia muy satisfactoria por los niños. Los pueden cuidar mediante gestos muy simples como ponerles música o cantarles una canción.

. Los celos naturales de las criaturas. Los celos son un estímulo de aprendizaje que tienen los niños y niñas hacia los adultos. Solo pueden ser negativos en caso que se repriman o se deprecie al infante por tener esta emoción.

. Varias formas de querer: Es importante que, durante el primer año de vida, se le den a los niños y niñas diferentes formas de afecto (abrazos, besos, etc.). En este sentido, el ser humano necesita muestras de afecto y contacto físico hasta el momento de su muerte. 
Las emociones están presentes en todos los acontecimientos de nuestra vida y, por lo tanto, también están relacionadas directamente con la muerte. Cuando los alumnos toman conciencia de la realidad de la muerte, las emociones sufren una gran revolución y se debe facilitar la expresión de los sentimientos para darles permiso para sentirlas. Por este motivo, si los adultos dejan que los infantes expresen sus emociones libremente, estos se podrán adaptar con más facilidad a las adversidades posteriores (Serra Llanas, 2014). Según la misma autora, si no se realiza de este modo, el niño no sabrá vivir las emociones que tiene y, posteriormente, cuando sufra una pérdida, puede desarrollar una desadaptación y la adversidad podrá provocarle desórdenes, tales como: aislamiento social, trastornos alimentarios, depresión, violencia, etc. Los humanos almacenamos y recordamos todas las experiencias que nos suceden y, a raíz de ellas, construimos un filtro o una red que determina los pensamientos y comportamientos posteriores (Griscom, 2000). Por ejemplo, si cuando éramos pequeños murió nuestro abuelo y lo vivimos como un acontecimiento misterioso y que nos daba miedo porque no nos explicaron que estar muerto no es el mismo que estar dormido, se pueden desarrollar fobias y miedos hacia la noche, la oscuridad, a lugar cerrados, etc. Es decir, si a los niños no se los explica aquello pueden sentir y se les da permiso para sentirlo, no se les acompaña en el duelo y no se les explica aquello que podrán ver (en el supuesto de que asistan a los ritos funerarios), la muerte puede acontecer un hecho perjudicial para el niño o niña. Tal y como sostiene Poch (2009) el hecho no saber y desconocer genera ansiedad, inseguridad e indefensión. Por estos motivos, es necesario que los infantes aprendan a exteriorizar sus emociones en relación a la muerte (Herrán, Rodríguez Herrero \& Yubero, 2019).

Arnaiz (2003), Ramos-Pla \& Camats (2019) y Ramos-Pla \& Camats (2018) afirma que la pedagogía preventiva o previa a la muerte es uno de los cimientos principales de la alfabetización emocional. El motivo que propone es muy claro, dado que la inteligencia emocional permite entender los procesos emocionales que se viven sobre la conciencia de la fragilidad, y, en definitiva, de la finitud. La comprensión y gestión de estas emociones permitirá a los alumnos poder hacer un trabajo del duelo positivo en el momento que muera un ser querido para ellos. 
Además, Kübler-Ross (1992), Poletti \& Dobbs (2004), Poch (2009), Esquerda \& Agustí (2010) abogan que se debe motivar los niños y adolescentes a manifestar su rabia, a llorar para expresar su dolor, a manifestar sus miedos y fantasías a alguien que los escuche y entienda, como podrían ser sus familias, docentes, amigos, etc. Se les debe transmitir que podemos comprender sus sentimientos mezclados, los cuales son muy humanos y todo el mundo los podemos tener. Para hacerlo, podemos emplear palabras como: "me gustaría que compartiéramos juntos tu dolor”, “¡no va bien quedárselo dentro!”. Además, se les debe hacerlos saber que tienen que enfrentar el reto de superar sus miedos para avanzar.

\subsection{Estrategias pedagógicas para afrontar la muerte}

La rabia es un sentimiento que se experimenta con mucha fuerza y que a menudo nos hace sentir muy mal (Díaz Teba, 2004; Poletti \& Dobbs, 2004; Esquerda \& Agustí, 2010; RamosPla, Gairín \& Camats, 2018; Ramos-Pla, 2018). Los infantes pueden canalizar este sentimiento empleando su cuerpo. Algunas actividades que proponen los autores citados son las siguientes:

. Todos los juegos y actividades que comporten ensuciarse o ensuciar (dibujar con pintura de dedos, pintura al temple, moldear barro, etc.).

- Todos los juegos que impliquen un ejercicio físico y una descarga de energía (correr, jugar a diferentes deportes, saltar, etc.). Un buen espacio para hacer estas actividades es la clase de educación física, el recreo escolar, así como en las tutorías.

. Las cosquillas.

. Todos los juegos y actividades donde se utilicen materiales que se puedan cortar, pinchar o golpear (perforar dibujos, recortar papeles, utilizar sierras y martillos infantiles, enganchar botones, etc.).

. Juegos de lanzamiento de objetos, como por ejemplo jugar al baloncesto.

. Romper o destruir cosas que se han construido previamente (como aplastar figuras hechas con plastilina, tumbar castillos de cartas, etc.). Se debe vigilar que el alumno no destruya ninguna cosa antes de haberla acabado. Destruir no es terapéutico si representa una reacción a la frustración (Díaz Teba, 2004). 
- Juegos que requieran un sentido competitivo. Sin embargo, se deberá vigilar que la competencia se establezca entre un niño y un adulto (y no entre dos niños) o entre equipos numerosos y equilibrados (mismo número de componentes y máxima igualdad de fuerzas y habilidades).

Los infantes y adolescentes en duelo se sienten diferentes, dado que el sentimiento de pérdida puede hacerlos pensar que no son tan importantes que los compañeros. Una buena forma de ayudarlos es potenciar su autoestima. Algunas de las estrategias que se pueden desarrollar son las siguientes (adaptado de Kübler-Ross, 1992; Ramos-Pla, Selfa \& Camats, 2016):

. Participar en juegos educativos que comporten aprender a hacer cosas nuevas, acordes con su edad.

. Confeccionar objetos por su habitación u ornamentos (carteles con su nombre, ornamentos por las camisetas, etc.).

. Hacer actividades creativas que impliquen diseñar y construir o inventar historias, cuentos, argumentos, etc.

. Dibujar, ya que es una importante vía de expresión.

. Elaborar comidas fáciles, adornar pasteles, preparar bocadillos, etc. Siempre se debe tener en cuenta la edad de los niños y adolescentes y estar alerta del fuego, cuchillos, etc.

No es aconsejable dejar que el niño o adolescente deje la tarea a medias si encuentra una pequeña dificultad y se desanima. Le tenemos que ayudar y motivar para que piense y reflexione las soluciones.

Es importante recordar que mediante el apoyo emocional y social al individuo que se encuentra en proceso de duelo, se potenciará la autoestima perdida con el impacto emocional (Lee, 1995; Masreal, 2007; Esquerda \& Agustí, 2010; Ramos-Pla, 2016a, 2016b). En definitiva, es ofrecer un espacio para expresar emociones, ser un mismo, reflexionar y repensar la muerte. 
Por los motivos que hemos ido mencionando hasta ahora, apostamos por la importancia que los profesionales de la educación potencien la educación emocional entre ellos mismos y los niños.

\section{Conclusiones}

Es necesaria una interrelación explícita entre Educación Emocional y pedagogía preventiva o previa a la muerte. El simple hecho de hacer visible la estrecha relación entre las dos temáticas, permite que los docentes se planteen la necesidad de una Educación para la muerte.

A lo largo de los últimos años ha proliferado en masa la literatura relacionada con la Educación Emocional y la Inteligencia Emocional, pero olvidando conectar con la pedagogía de la muerte (sobre todo en su vertiente previa o preventiva). Si bien es cierto que la muerte continúa siendo un tabú social (con la consecuencia directa de contaminar el pedagógico), se deben emplear todos los esfuerzos en normalizarla mediante la Educación Emocional.

Por último, no se debe olvidar que los centros educativos no solo forman en contenidos meramente curriculares, sino que también se educa a los ciudadanos de un mundo globalizado (Ramos-Pla, 2021; Ramos-Pla, 2020c). Por ello, es importante incluir todas aquellas competencias vinculadas a la Educación Emocional y a la pedagogía preventiva o previa a la muerte que permiten el desarrollo integral del alumnado. Este hecho, posibilitará su preparación para futuras muertes y/o pérdidas, aprendiendo a gestionar sus emociones. 


\section{Referencias}

Aradilla, A. (2004). La muerte una pedagogía emocional. MONITOR EDUCADOR, 104, 1316

Arnaiz, V. (2003). Diez propuestas para una pedagogía de la muerte. Aula de Innovación Educativa, 122, 59-61

Asensi, J. (2012). Pedagogía de la muerte a través del cine. Indivisa, Boletín de Estudios e Investigación, 13, 218-220

Benlloch, M.J., Vazquez, V., Boluda, J., y Garcia Bataller, E. (2015). APS como metodologia para trabajar la muerte y el duelo en futuros educadores. Pedagogía ante la muerte. Reflexiones e interpretacions en perspectives històrica y filosófica, 21-27

Bisquerra, R. (2003). Educación emocional y competencias básicas para la vida. Revista de Investigación Educativa, 21(1), 7-43

Bisquerra, R. (2006). Orientación psicopedagògica y educación emocional. Estudios sobre Educación, 11, 9-25

Bisquerra. R. (2016). Competències emocionals. Barcelona: Rafael Bisquerra.

Campillo Ruiz, E., y Ruiz Arriaza, J. (2017). Educar para la muerte a través de la didáctica de la literatura. Publicaciones didácticas, 82, 648-653

Carmelo, A. y Comas, L. (2014). ¿Existe la muerte? Ciencia, vida y trascendencia. Plataforma Editorial 
Chau, L. Y., Cheung, W.S., y Ho, S.M.Y. (2018). Change in death metaphors among university students in Hong Kong from 2004 to 2016. Death Studies, 1, 1-9

Colomo, E. (2016). Pedagogía de la Muerte y proceso de duelo. Cuentos como recurso didáctico. REICE Revista Iberoamericana sobre Calidad, Eficacia y Cambio en Educación, $14(2), 1-15$

Colomo, E., y Cívico, A. (2018). La necesidad de formación del profesorado en pedagogía de la muerte. Revista Electrónica Interuniversitaria de Formación del Profesorado, 21(1), 8394

Corr, C.A. (2016). Teaching about life and líving in courses on death and dying. OMEGA, 72(2), 174-187

Cortina, M., Herrán, A., y Nolla, A. (2009). Propuestas para anticiparse al duelo. Cuadernos de Pedagogía, 388, 68-72

del Arco, I., Flores, O. y Ramos-Pla, A. (2021). Estructural model for determining the factors that affect the quality emergency teaching, accoding to the perception of the student of the first university courses. Sustainability, 13(5), 2945. https://doi.org/10.3390/su13052945

Darder, P., y Bach, E. (2006). Aportacions para repensar la teoria y la pràctica educatives desde las emociones. Revista Teoría de la Educación, 18, 55-84

Díaz Teba, I. (2004). ¿I ara, on és? Com ajudar els nens i els adolescents a entendre la mort. Oxigen Viena

Dollenz, E. (2003). Duelo normal y patológico. Consideracions para el Trabajo en atención primaria de salud. Universidad de Santiago de Chile.

Esquerda, M., y Agustí, A. M. (2010). El nen i la mort. Acompañar els infants $i$ adolescents en la pèrdua d'una persona estimada. Pagès Editors. 
Extremera, N., y Fernández-Berrocal, P. (2004). El papel de la inteligencia emocional en el alumnado: evidencias empíricas. REDIE, Revista Electrónica de Investigación Educativa, $6(2), 1-17$

Gardner, H. (2010). La inteligencia reformulada: las inteligencias múltiples en el siglo XXI. Paidós.

Gao, M., y Slaven, M. (2017). Best practices in children's bereavement: A qualitative analysis of needs and services. Journal of Pain Management, 10(1), 119-126

Gill, S.A. (2016). The predictive relationship between emotional expressiveness and discussing death with children: an exploratory study with online college parents. (Tesis doctoral). Walden University, Minneapolis, Minnesota

Girardi, N., y San Gil, M.; Santillán, S. (2009). ¿Qué piensan los ninos acerca de la muerte y qué actitudes toman los adultos frente a esto? e-Ecleston. Temes de Educación Infantil, 12(2), 1-7

Goleman, D. (1995). Intelgencia Emocional. Kairós

Gómez Ruiz, S. (2013). “Sí, me he sentido triste, pero no se lo puedo decir”: la reflexividad etnográfica en la investigación sobre emociones de la muerte con niños y niñas de Sumapaz en contexto de (post)conflicto. Antípoda, Revista de Antropología y Arqueología, 16, 135-156 Griscom, C. (2000). Sanar las emociones. Luciérnaga.

Guil, R., Gil-Olarte, P., Mestre, J.M., y Nuñez, I. (2006). Intel-ligència Emocional y adaptación socioescolar. REME. Revista Electrónica de Motivación y Emoción, 9(22), 21-32 Head, B.A., y Smith, L.C. (2016). Use of contemporary firlm as a mèdium for teaching an online death and grief course. Journal of Social Work in End-of-Life and Palliative Care, 12, 195-213 
Herrán, A. (2015). Pedagogía radical e inclusiva y educación para la muerte. FahrenHouse

Herrán, A. (2017). Relfexiones pedagógicas desde el enfoque radical e inclusivo de la formación. FahrenHouse

Herrán, A., Rodríguez Herrero, P, y Yubero, V. M. (2019). ¿Está la muerte en el currículo español? Revista de Educación, 385, 201-226. https://doi.org/10.4438/1988-592X-RE-2019$385-422$

Huisman-Perrin, E. (2003). La mort explicada a la meva filla. Editorial Empúries

Kübler-Ross, E. (1992). Todo final es un luminoso principio. Sirpus Edición

Lantieri, L. (2009). Inteligencia emocional infantil y juvenil. Ejercicios para cultivar la Fortaleza interior en niños y jóvenes. Santillana

Latorre, J. M., y Montañés, J. (2004). Ansiedad, inteligencia emocional y salud en la adolescencia. Ansiedad y Estrés, 10(1), 111-125

Lee, C. C. (1995). Culturally responsive school counselors and programs: adressing the needs of all students. Professional School Counseling, 4(4), 257

Mantegazza, R. (2006). La muerte sin máscara. Experiencia del morir y educación para la despedida. Herder

Martínez-Heredia, N., y Bedmar, M. (2020). Impacto de la producción científica acerca de la educación para muerte: Revisión bibliométrica en Scopus y Web of Science. Revista Iberoamericana de Educación, 82(2),65-79. https://doi.org/10.35362/rie8223553

Mèlich, J-M. (2011). Filosofía de la finitud. Herder Editorial 
Masreal, F. (2007). Conviure amb la depressió. Mina

Mestre Navas, J.M., y Fernández Berrocal, P. (2007). Manual de la Inteligencia Emocional. Pirámide

Morell-Velasco, C., Fernández-Alcántara, M., Hueso-Montoro, C., Montoya-Juárez, R. (2018). Teachers' perception of grief in primary and secondary school students in Spain: Children's responses and elements which facilitate or hinder the grieving process. Journal of Pediatric Nursing, 1-8 <https://doi.org/10.1016/j.pedn.2019.12.016>

Park, O., Lee, Y.K., Kim, Y., y Smith-Stonen, M. (2016). The effect on insight based death meditation on death anxiety and quality of life. International Journal of Existencial Psychology \& Psychotherapy, 6(1), 1-10

Pedrero-García, E. (2020). Pedagogía de la muerte: propuesta de normalización en el sistema educativo espanyol. Espacios, 41(4), 25-37

Peniston, H. (2015). The importance of "death education" in family life. National council of family relations, 11(1), 15-18

Poch, C. (2009). La muerte nunca falla. Un doloroso descubrimiento. Niberta

Poletti, R., y Dobbs, B. (2004). Cómo crecer a través del duelo. Barcelona: Ediciones Obelisco

Ramos-Pla, A. (2021). Aprendizaje organizativo e informal, reseña de Aprendizaje organizativo e informal, de Gairín, J., Rodríguez-Gómez, D., Revista de Educación, 392, 231232. 
Ramos-Pla, A. y Gairín, J. (2020). Estudio de la pedagogía preventiva sobre la muerte: reto y oportunidad. Espacios, 41(42), 52-65. https://doi.org/10.48082/espacios-a20v41n42p05

Ramos Pla, A., Gairín, J. y Camats, R. (2020). Percepciones educativas en relación a la pedagogía preventiva de la muerte. Espacios, 41(4), 4-12.

Ramos-Pla, A. (2020a). Fundamentos y evolución de la pedagogía preventiva sobre la muerte a través de la filosofía. Revista Educación de la Universidad Pontificia de Perú, 29(56), 193210. https://doi.org/10.18800/educacion.202001.009

Ramos-Pla, A. y Selva, L. (2020). DOTS-educa: 10 meses, 10 retos. Dykinson

Ramos-Pla, A. (2020b). sanaMENTE. En A. Ramos-Pla \& L. Selva Pareja (Ed.), DOTS educa, 10 meses 10 retos (pp. 34-37). Madrid: Editorial Dykinson

Ramos- Pla, A. (2020c). Análisis de la situación actual de la pedagogía preventiva sobre la muerte: currículum y formación del profesorado. Ensayos. Revista de la Facultad de Educación de Albacete, 35(2), 221-233. https://doi.org/10.18239/ensayos.v35i2.2358

Ramos-Pla, A. (2020d). Pedagogía de la muerte en tiempos de la pandemia del COVID-19. [Comunicación]. VII Congreso Internacional de Educación, Méjico.

Ramos-Pla, A. (2020e). Pedagogía de la muerte y educación para la salud: relación y posibilidades [Comunicación]. IV Congreso Internacional de Investigación e Innovación Educativa, Méjico.

Ramos Pla, A. y Camats, R. (2019). Consideraciones generales respecto la necesidad de una pedagogía sobre la finitud humana en la educación formal. Estudio de caso. EDUCAR, 55(1) 273-290. https://doi.org/10.5565/rev/educar.950

Volumen 2. Número 2. Julio - Diciembre 2022 
Ramos Pla, A. y Camats, R. (2018). Fundamentos para una pedagogía preventiva sobre la muerte en la escuela. Revista Complutense de Educación, 29(2), 527-538. https://doi.org/10.5209/RCED.53448

Ramos Pla, A., Gairín, J. y Camats, R. (2018). Principios prácticos y funcionales en situaciones de muerte y duelo para profesionales de la educación. REICE Revista Iberoamericana sobre Calidad, Eficacia y Cambio en Educación, 16(1), 21-33. https://doi.org/10.15366/reice2018.16.1.002

Ramos Pla, A. (2018). Estudio de las prácticas educativas en relación a la pedagogía preventiva sobre la muerte. En I. del Arco y P. Silva. Tendencias nacionales e internacionales en organización educativa: entre la estabilidad y el cambio (pp.16-49). Madrid: Wolters Kluwer

Ramos-Pla, A. (2017). ¿Formar al profesorado en pedagogía de la muerte? Cuadernos de Pedagogía, 483, 82-85

Ramos-Pla, A. (2016a). El psicopedagogo como guía en los procesos de muerte y duelo infantil. Revista Àmbits de Psicopedagogia i Orientació, 45(2), 1-8

Ramos-Pla, A., Selfa Sastre, M. y Camats i Guàrdia, R. (2016). Cómo afrontar la muerte y el duelo en el aula. Cuadernos de Pedagogía, 468, 76-80

Ramos-Pla, A. (2016b). Investigación cualitativa respecto la importancia del diseño de una propuesta para el desarrollo de un programa pedagógico sobre la finitud humana en centros de Educación Primaria. En J. L. Bernal. Globalización y organizaciones educativas (pp. 247256). Zaragoza: Universidad de Zaragoza 
Ransanz, E. (2015). La muerte en el desarrollo de la madurez personal del educando. Pedagogía ante la muerte. Reflexiones e interpretacions en perspectives històrica y filosófica, $161-167$

Rodríguez Herrero, P., Herrán, A., y Cortina, M. (2015). Educar y vivir teniendo en cuenta la muerte. Reflexiones y propuestas. Ediciones Pirámide

Salovey, P., y Mayer, J.D. (1990). Emotional intelligence. Imagination, Cognition, and Personality, 9, 185-211.

Salovey, P., Mayer, J., y Caruso, D. R. (2008). Emotional Intelligence. New ability or eclectic traits? American Psichologist, 63(6), 503-517

Serra Llanas, X. (2014). ¿I jo, també em moriré? Com es pot ajudar els infants i els joves a conviure amb la pèrdua i la mort de qui estimem. Columna

Torralba, F. (2008). Planta cara a la mort. Ara Llibres, S.C.C.L

Valenzano, N. (2015). Educare alla morte per educare allà caducitat e allà vita. Dins de Pedagogía ante la muerte. Reflexiones e interpretacions en perspectives històrica y filosófica, 205-207

Vallés, A., y Vallés, C. (2003). Psicopedagogía de la Inteligencia Emocional. Editorial Promolibro

Yang, S., y Park, S. (2017). A sociocultural approach to children's perceptions of death and loss. OMEGA, 1, 1-25 